\title{
Qual o Seu Diagnóstico?
}

\section{Lesões Bolhosas Palmares no Idoso}

Santiago $L^{1}$, Cardoso JC', Tellechea $\mathrm{O}^{\prime}$

'Departmento de Dermatovenereologia, Centro Hospitalar e Universitário de Coimbra, Coimbra, Portugal

PALAVRAS-CHAVE - Dermatopatias Vesiculobolhosas; Dermatoses da Mão/diagnóstico; Idoso; Penfigoide Bolhoso/diagnóstico.

\section{Dermatology Quiz}

\section{Hand Bullous Lesions in the Elderly}

KEYWORDS - Aged; Hand Dermatoses/diagnosis; Pemphigoid, Bullous/diagnosis; Skin Diseases, Vesiculobullous.

\section{CASO CLÍNICO}

Relatamos o caso de um homem de 79 anos com história de lesões vesiculosas e bolhosas pruriginosas localizadas a nível palmar bilateral. Referia aparecimento há cerca de 2 anos, com resposta parcial a dermocorticóides de baixa-média potência e carácter recidivante. Ao exame objetivo apresentava múltiplas lesões vesiculobolhosas tensas com base eritematosa, a maioria em fase erosiva (Fig. 1). A nível plantar referia lesões no passado, que não apresentava no momento da consulta. Não apresentava outras alterações cutâneas ou mucosas.

Foi enviada amostra para cultura de fungos que foi negativa. O estudo histopatológico de biópsia cutânea mostrou descolamento bolhoso subepidérmico com extensa reepitilização subjacente associado a infiltrado linfomononucleado perivascular rico em eosinófilos (Fig. 2). A imunofluorescência direta (IFD) de área perilesional mostrou depósitos lineares de C3 e lgG ao nível da junção dermo-epidérmica. Foram detectados anticorpos circulantes para BP180 (122 U/mL, normal >20 U/mL) por ELISA. Foi iniciado tratamento com prednisolona $30 \mathrm{mg} / \mathrm{dia}$ em desmame durante 2 semanas associado a dermocorticóide de alta potência com resolução das lesões e sem formação de novas bolhas em dois anos de follow-up.
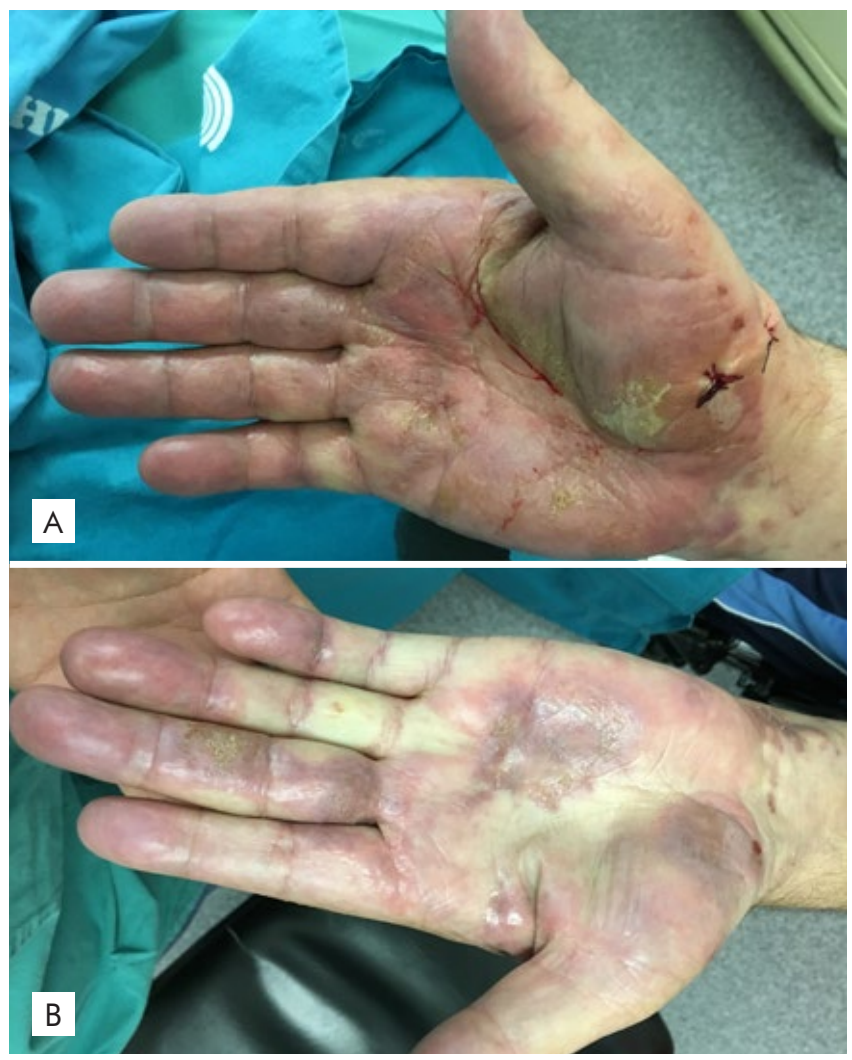

Figura 1 - Lesões vesiculobolhosas com base eritematosa associadas a erosões a nível palmar bilateral.
Correspondência: Luís Santiago

Serviço de Dermatovenereologia - Centro Hospitalar e Universitário de Coimbra

Praceta Prof. Mota Pinto - 3000-075 Coimbra, Portugal

E-mail: lvisgsantiago2@gmail.com

DOI: https://dx.doi.org/10.29021/spdv.77.2.1061
Recebido/Received

9 Abril/April 2019

Aceite/Accepted

1 Maio/May 2019 


\section{Qual o Seu Diagnóstico?}

\section{DIAGNÓSTICO}

\section{PENFIGÓIDE BOLHOSO DESIDROSIFORME}

O penfigóide bolhoso (PB) é uma dermatose bolhosa autoimune subepidérmica associada à presença de anticorpos contra hemidesmossomas da membrana basal, na maioria dos casos as proteínas BP 180-kDa transmembranar e 230$\mathrm{kDa}$ intracelular. ${ }^{1}$ Rongioletti et al ${ }^{2}$ descreveram três variantes de PB localizado: o tipo Brunsting-Perry com lesões bolhosas cicatriciais no pescoço/cabeça; um segundo tipo com lesões bolhosas na região pré-tibial; e a variante desidrosiforme palmoplantar. Recentemente outras variantes foram descritas: vulvar, peristomal, umbilical, do coto de amputação, do membro paralisado e em locais de radioterapia. ${ }^{3}$

O penfigóide bolhoso desidrosiforme (PBD) é uma variante rara de PB localizado descrita em 1979 por Levine et al. ${ }^{4}$ Clinicamente apresenta-se como uma erupção vesiculoboIhosa persistente semelhante a um eczema desidrótico, por vezes, com carácter hemorrágico.

$\mathrm{Na}$ literatura foram descritos vários casos de envolvimento palmoplantar com apresentações clínicas diferentes. Caldora et a ${ }^{5}$ dividiram estes casos em 3 grupos: grupo 1 caracterizado exclusivamente por erupção vesiculobolhosa palmoplantar; grupo 2 com erupção palmoplantar prévia a envolvimento clássico por PB do restante tegumento em meses/anos; e grupo 3 com PB desidrosiforme concomitante com PB generalizado. Este último grupo ocorre em cerca de $28 \%$ dos casos de PB clássico, ${ }^{6}$ sendo mais comum na população pediátrica. ${ }^{7,8}$ Considerando que o PB localizado

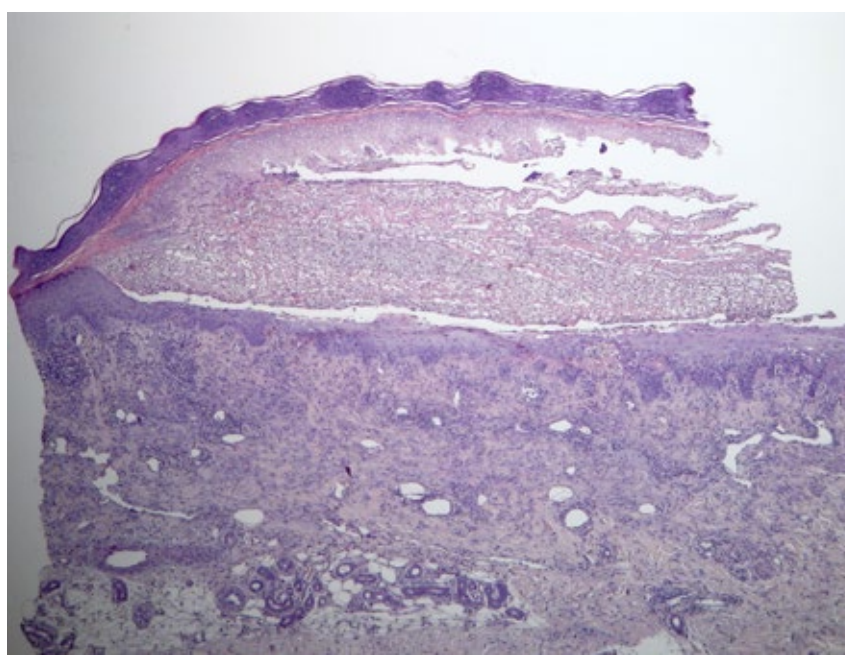

Figura 2 - Imagem histopatológica de biópsia palmar que mostra descolamento bolhoso subepidérmico com reepitelização subjacente acompanhada de infiltrado mononucleado perivascular que se observa igualmente no conteúdo do descolamento bolhoso (H\&E, X200). por definição compreende lesões confinadas a determinada área cutânea com ou sem posterior envolvimento sistémico, apenas o grupo 1 (e provavelmente o 2) poderão ser considerados verdadeiros casos de PBD.

A prevalência do PBD é desconhecida, estando até ao momento descritos na literatura 35 casos: 12 com localização exclusiva a nível palmoplantar e 7 com posterior generalização pelo tegumento. Contudo, provavelmente esta patologia é subdiagnosticada. ${ }^{5}$

No PBD as características histopatológicas e a imunofluorescência directa são consistentes com PB, ${ }^{9}$ dada a presença de anticorpos anti-membrana basal. Contudo, o mecanismo subjacente ainda não é compreendido. Uma explicação sugerida é a da existência de um processo inflamatório inicial ${ }^{4,6,10}$ (por exemplo, tinea pedis, queimaduras térmicas, trauma repetido, dermatite de contacto/irritativa ou radioterapia) que poderá expor os antigénios membranares apenas nessas localizações. Outro factor que poderá estar relacionado é a maior expressão de antigénio $\mathrm{BP}$ a nível plantar. ${ }^{11}$

As manifestações clínicas de PBD são difíceis de distinguir de patologias benignas mais frequentes como desidrose ou tinea manuum bolhosa, o que pode atrasar o diagnóstico.

Concluímos que na presença de dermatite desidrosiforme palmoplantar em idade geriátrica, recorrente, severa, hemorrágica ou resistente ao tratamento com dermocorticóides de elevada potência, o diagnóstico de PBD deve ser ponderado e deverá ser devidamente suportado por análise histopatológica e IFD.

Conflitos de interesse: Os autores declaram não possuir conflitos de interesse.

Suporte financeiro: $O$ presente trabalho não foi suportado por nenhum subsídio ou bolsa.

Confidencialidade dos dados: Os autores declaram ter seguido os protocolos do seu centro de trabalho acerca da publicação dos dados de doentes.

Direito a privacidade e consentimento escrito: Os autores declaram que pediram consentimento para usar as imagens no artigo.

Conflicts of interest: The authors have no conflicts of interest to declare.

Financing Support: This work has not received any contribution, grant or scholarship.

Confidentiality of data: The authors declare that they have followed the protocols of their work center on the publication of data from patients.

Privacy policy and informed consent: The authors declare that have the written informed consent for the use of patient's photos in this article.

Proveniência e revisão por pares: Não comissionado; revisão externa por pares

Provenance and peer review: Not commissioned; externally peer reviewed 


\section{Qual o Seu Diagnóstico?}

\section{REFERÊNCIAS}

1. Chimanovitch I, Hamm H, Georgi M, Kroiss M, Stolz W, Apitz C, et al. Bullous pemphigoid of childhood: autoantibodies target the same epitopes within the NC16A domain of BP180 as autoantibodies in bullous pemphigoid of adulthood. Arch Dermatol. 2000; 136:527-32.

2. Rongioletti F, Parodi A, Rebora A. Dyshidrosiform pemphigoid: report of an additional case. Dermatologica. 1985; 17:84-5.

3. Bernard P, Borradori L. Pemphigoid group. In: Bolognia JL, Schaffer JV, Cerroni L. Dermatology. London: Mosby Elsevier; 2018.

4. Levine N, Freilich A, Barland P. Localized pemphigoid simulating dyshidrosiform dermatitis. Arch Dermatol. 1979;1 15:320-1.

5. Caldarola G, Fania L, Cozzani E, Feliciani C, De Simone C. Dyshidrosiform pemphigoid: a well-defined clinical entity? Eur J Dermatol. $2011 ; 21: 112-3$. doi: 10.1684/ ejd.2010.1172.

6. Barth JH, Venning VA, Wojnarowska F. Palmo-plantar involvement in auto-immune blistering disorders - pemphigoid, linear $\lg A$ disease and herpes gestationis. Clin Exp Dermatol. 1988; 13:85-6.

7. Ferreira BR, Vaz AS, Ramos L, Reis JP, Gonçalo M. Bullous pemphigoid of infancy - report and review of infantile and pediatric bullous pemphigoid. Dermatol Online J. $2017 ; 16 ; 23$

8. Ramos L, Tellechea O, Moreno A, Martins L, Neves N, Januário $G$, et al. Childhood bullous pemphigoid: Report of 2 cases. J Eur Acad Dermatol Venereol. 2016; 30:330-2. doi: 10.1111/idv.12724.

9. Soh H, Hosokawa H, Miyauchi H, Izumi H, Asada Y. Localized pemphigoid shares the same target antigen as bullous pemphigoid. Br J Dermatol. 1991; 125:73-5.

10. Knees-Matzen S, Proksch E, Meigel WN. Dyshidrosiform bullous pemphigoid: Trigger factors. J Eur Acad Dermatol Venereol. 1996;7:257-62.

11. Hamm G, Wozniak KD. Bullous pemphigoid antigen concentration in normal human skin in relation to body area and age. Arch Dermatol Res. 1988;280:416-9. 